\title{
Mixing Lengths of Reacting and Nonreacting Coaxial Injectors in a Laboratory Rocket Combustor
}

\author{
S. Alexander Schumaker* and James F. Driscoll ${ }^{\dagger}$ \\ The University of Michigan, Department of Aerospace Engineering, 1320 Beal Avenue, Ann Arbor, MI 48109
}

\begin{abstract}
Stoichiometric mixing lengths are obtained for coaxial jets with and without combustion in a rocket fuel injector configuration. With a center jet of oxidizer (oxygen or air) and a surrounding annular jet of hydrogen these flames are relatively short resulting in the mixing primarily occurring in the near field. This produces a different scaling than the far field analysis of a turbulent jet flame, where a fuel jet is injected into a coflow of oxidizer. Stoichiometric mixing lengths $\left(L_{S}\right)$, defined as the distance along the centerline where the stoichiometric condition occurs, were measured using Planar Laser Induced Fluorescence (PLIF). Acetone seeded into the center jet along with quantitative acetone PLIF allowed the direct measurement of the average and instantaneous mixture fraction fields for a range of velocity and density ratios. For hydrogen-oxygen and hydrogen-air coaxial jet flames, $L_{S}$ was measured from the $\mathrm{OH}$ radical field obtained using $\mathrm{OH}$ PLIF. Due to the inverse natural of these flames and since all cases were run fuel rich, $\mathrm{OH}$ forms thin layers near the stoichiometric contour. Using strained laminar flame calculations from Chemkin and correcting for absorption and quenching effects, the stoichiometric value of the $\mathrm{OH}$ signal was related to the peak signal. In nonreacting cases the use of a nondimensional momentum ratio collapses the nonreacting coaxial jet data. To account for the effect of heat release in reacting cases the equivalence principle of Tacina and Dahm is utilized to produce an equivalent outer gas density to create a new effective momentum ratio. This method is found to slightly under predict the effect of heat release for both hydrogen-oxygen and hydrogen-air turbulent coaxial jet flames.
\end{abstract}

\section{Introduction}

One common method to mix fuel and oxidizer in rocket engines and industrial furnaces is to employ a coaxial jet injector, due to both the simplicity of the geometry and the rapid mixing that it provides. In these applications the denser oxidizer often is the inner jet which is surrounded by an annulus of lower density fuel. One advantage of such a design is that strong oxidizers are encased in an envelope of fuel which keeps the oxidation of combustion chamber walls to a minimum. Also, such a design with a strong oxidizer results in a relatively short flame where mixing occurs primarily in the near field.

A number of studies have been conducted that examine the near field region of nonreacting coaxial jets. ${ }^{1-5}$ From these studies, two important governing parameters that effect near field mixing are the velocity and density ratio:

$$
r_{u}=u_{e} / u_{i}, \quad S=\rho_{e} / \rho_{i}
$$

where e denotes the outer jet and i denoted the inner jet. Villermaux and Rehab ${ }^{2}$ studied water jets and used a simple entrainment argument to show that the potential core length is proportional to $r_{u}$ and hypothesized that the effects of variable density jets could be accounted for by replacing $r_{u}$ with the square root of the momentum ratio $(M)$, which is:

$$
M=S r_{u}^{2}
$$

\footnotetext{
*Graduate Student, Aerospace Engineering Department, The University of Michigan, Ann Arbor, MI, Member

${ }^{\dagger}$ Professor, Aerospace Engineering Department, The University of Michigan, Ann Arbor, MI, Fellow
} 
Work by Favre-Marient and Schetti ${ }^{3}$ showed that the potential core length is proportional to $M^{1 / 2}$ for variable density nonreacting jets up to a momentum ratio of approximately 50 where recirculation in the inner jet starts to occur. A nonreacting flow study by the present authors reported that the centerline values of $L_{S}$ (the distance along the centerline where stoichiometric conditions occur) for cases of hydrogenoxygen and methane-oxygen mixing are also proportional to $M^{1 / 2}{ }^{6}$ Whereas a majority of these studies cite combustion applications as a main reason for studying the flow field of coaxial jets, very few studies have actually investigated reacting flows. ${ }^{7-11}$ The few studies of reacting coaxial jet flames have not quantified stoichiometric mixing lengths $\left(L_{S}\right)$ or compared values of $L_{S}$ for reacting and nonreacting conditions. Both are done in the present work while systematically varying the parameters known to be important to coaxial jet mixing, namely $r_{u}$ and $S$.

Quantitative acetone PLIF was used in nonreacting cases to obtain the instantaneous and average mixture fraction field from which the stoichiometric contour and hence $L_{S}$ was obtained. For the reacting cases qualitative $\mathrm{OH}$ PLIF was employed. Counterflow flame calculations were made using Chemkin and the method of Barlow and Colligion ${ }^{12}$ was used to correct for Boltzmann fraction and quenching in the PLIF signal, to provide a criteria for the relationship between the peak $\mathrm{OH}$ contour and the stoichiometric contour. Based on this analysis, thresholding was used on the thin $\mathrm{OH}$ structures found in the instantaneous PLIF images to produce an instantaneous flame front from which averaged images were produced and values of $L_{S}$ were calculated. To relate the nonreacting and reacting values of $L_{S}$, the equivalence principle suggested by Tacina and Dahm ${ }^{13,14}$ was utilized. This method relates the reacting flow field to the simple mixing of two nonreacting fluids by assuming a new "equivalent" gas density of the outer fluid. The equivalence principle has been used effectively to account for heat release effects in momentum dominated turbulent axi-symmetric and planar jets and turbulent shear layers, however it had not previously been applied to turbulent coaxial jet flames or oxygen enriched combustion. The present paper continues on the work to appear in the $32^{\text {nd }}$ International Symposium on Combustion by adding results obtained for hydrogen-air reacting cases and additional data obtained at one atmosphere. ${ }^{15}$

\section{Coaxial Turbulent Jet Flame Mixing Length Model}

The ability to predict the lengths of turbulent coaxial diffusion flames is important because it helps determine the sizing of the combustor or furnace and the heat transfer to that apparatus. For the fueloxidizer combinations common in rockets and industrial furnaces the stoichiometric mixture fraction based on inner jet fluid $\left(f_{s}\right)$ is relatively large; $f_{s}=0.97$ for hydrogen-air, $f s=0.89$ for hydrogen-oxygen and $f s=0.80$ for methane-oxygen. Thus the distance to mix to stoichiometric on the centerline $\left(L_{S}\right)$ is typically only 20 percent larger then the jet core length, hence it is determined primarily by near field mixing and not far field mixing. A coaxial jet differs from a single jet and a coflowing jet because the near field is characterized by the interaction of two shear layers. The outer mixing layer in Fig. 1 lies between the ambient fluid and the outer jet and the inner mixing layer lies between the inner jet and the outer jet. In the case of a closed combustor the ambient fluid is a combination of combustion products and any excess fuel or oxidizer.

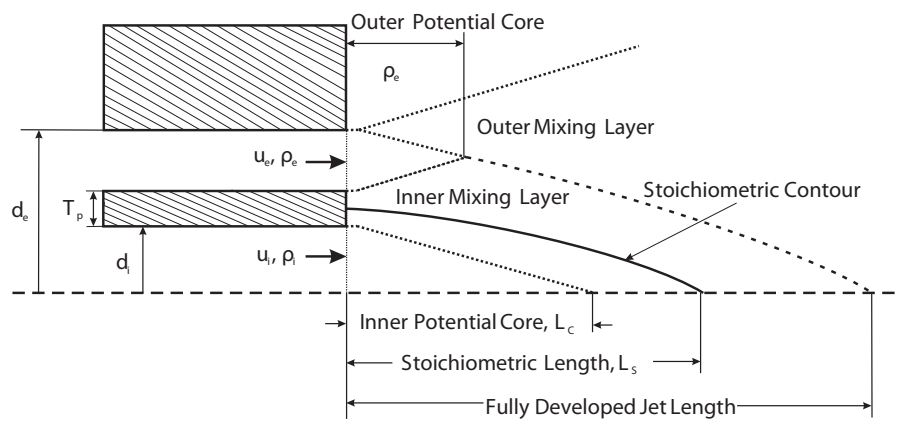

Figure 1. Schematic of coaxial jet injector and the near field mixing layers.

One method to predict $L_{S}$ in nonreacting coaxial jets is based on a global mass entrainment argument. $^{2}$ Assuming a cylindrical shape with a constant entrainment velocity $\left(u_{e e}\right)$ on the surface, the length of a cylinder required to entrain a stoichiometric amount of outer jet fluid is $L_{S}$. Therefore the ratio of the 
volume per second of inner jet fluid through the cylinder to the volume per second of fluid entrained across the cylindrical surface is

$$
\left(\pi u_{i}\left(d_{i} / 2\right)^{2}\right) /\left(\pi d_{i} L_{S} u_{e e}\right)=X_{S},
$$

where $X_{S}$ is the mole fraction of inner jet fluid in a stoichiometric mixture. Data for variable density jets indicate that: ${ }^{3,16}$

$$
u_{e e} \sim S^{1 / 2} u^{\prime},
$$

where $u^{\prime}$ is the turbulent velocity fluctuations.

For the case where $r_{u}>>1$, previous studies show that the turbulence level is controlled by the outer jet velocity so $u^{\prime} \sim u_{e}{ }^{1,2}$ Inserting Eq. (4) into Eq. (3) and solving for $L_{S}$ yields the fundamental scaling relation:

$$
\frac{L_{S}}{d_{i}}=C_{1}\left(M^{1 / 2} X_{S}\right)^{-1},
$$

where $C_{1}$ is a scaling constant and $M$ is the momentum ratio defined by Eq. (2). It is noted that $X_{S}$ is related to the stoichiometric mixture fraction $\left(f_{S}\right)$ by:

$$
X_{S}=\left(\frac{M W_{i}}{M W_{e}}\left(f_{S}^{-1}-1\right)+1\right)^{-1} .
$$

When combustion is considered, the dominant effect of heat release in nonpremixed combustion in the absence of buoyancy is to decrease the density of the gas. The decrease in the density results in a reduction in the mass entrainment rate which lengthens $L_{S}$. To apply the above cold flow scaling relation (Eq. (5)) to reacting flows, the method of Tacina and $\mathrm{Dahm}^{13}$ was considered to account for the decrease in density. Tacina and Dahm related the reacting flow field to the simple mixing of two fluids with the outer fluid having an effective temperature $\left(T_{e f f}\right)$ and a corresponding (low) effective density $\left(\rho_{\text {eff }}\right)$.

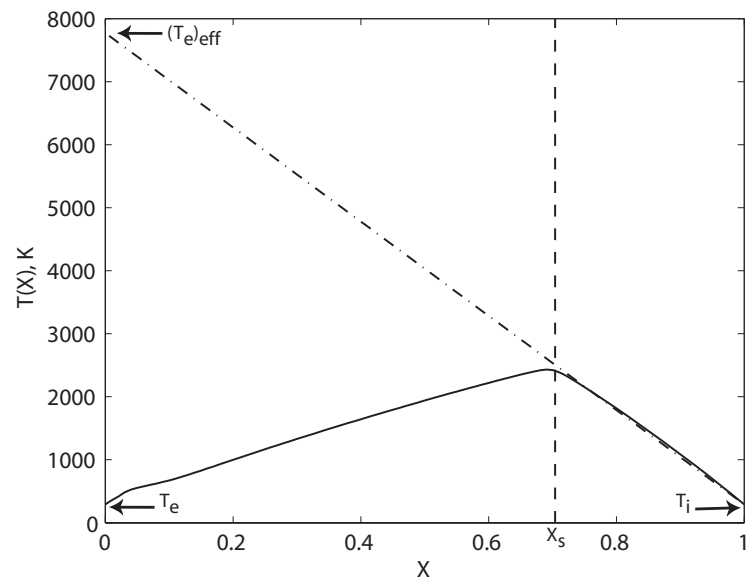

(a)

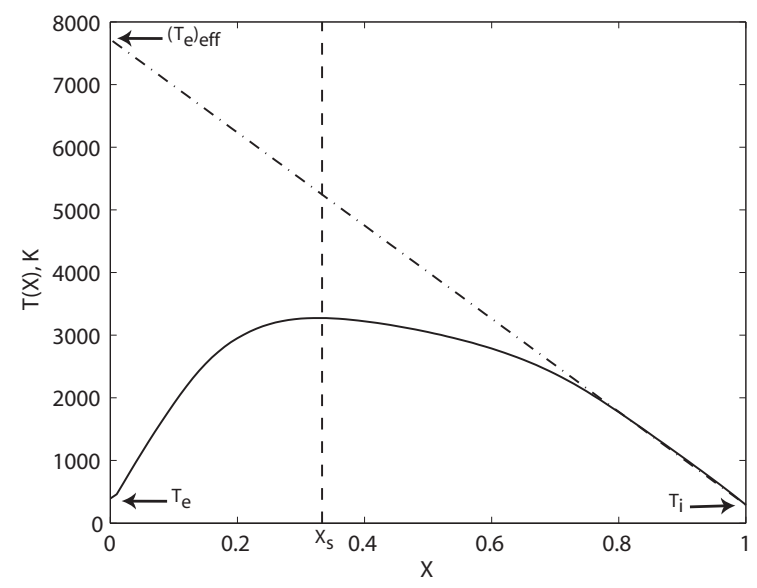

(b)

Figure 2. Equilibrium temperature state relation for a) hydrogen-air and b) hydrogen-oxygen where $X$ is the oxidizer mole fraction. $\left(T_{e}\right)_{e f f}$ is calculated from the equivalence principle of Tacina and Dahm. ${ }^{13}$

Figure 2 graphically illustrates how the method of Tacina and Dahm ${ }^{13}$ is applied to hydrogen-air and hydrogen-oxygen chemistry. The solid line is the equilibrium temperature state relation; $\mathrm{T}$ is plotted against the oxidizer mole fraction $(\mathrm{X})$. The dash-dot line is tangent to the solid curve at $X=1$; its $\mathrm{y}$-intercept is the effective outer gas temperature $\left(\left(T_{e}\right)_{e f f}\right)$. When this analysis is applied to hydrogen-oxygen $\left(T_{e}\right)_{e f f} / T_{e}=$ 26.2 and for hydrogen-air $\left(T_{e}\right)_{e f f} / T_{e}=26.5$. In a similar manner the effective molecular weight ratio $\left(\left(M W_{e}\right)_{e f f} / M W_{e}\right)$ is calculated to be 3.6 and 7 for hydrogen-oxygen and hydrogen-air respectively. Using the perfect gas law the normalized outer effective density is 


$$
\frac{\left(\rho_{e}\right)_{e f f}}{\rho_{e}}=\left(\frac{T_{e}}{\left(T_{e}\right)_{e f f}}\right)\left(\frac{\left(M W_{e}\right)_{e f f}}{M W_{e}}\right) .
$$

For hydrogen-oxygen combustion with an injection gas temperature of $293 \mathrm{~K}$ and injection pressure of 4.4 atmospheres, a normalized outer effective density of 0.136 was calculated, whereas for hydrogen-air combustion at a chamber pressure of 4.8 atmospheres a value of 0.264 was found. The outer effective density can be used to define an effective momentum ratio,

$$
M_{e f f}=S r_{u}^{2}\left(\rho_{e}\right)_{e f f} / \rho_{e}
$$

The present measurements were used to assess whether $M_{\text {eff }}$ collapses the reacting and nonreacting results to a single curve when substituted into Eq. (5) for $M$.

\section{Experimental Setup}

Experiments were conducted in the Michigan Single Element Injector Experiment shown in Fig. 3. ${ }^{6,17}$ The modular design allows a window section to be moved to any location in the combustion chamber and for the combustion chamber length to be varied by the addition or removal of spacer sections. In the $50.8 \times 50.8$ $\mathrm{mm}$ square chamber with rounded corners pressures up to 10 atmospheres can be achieved. The confining walls have minimal effect on the near field mixing as confirmed by measurements; ${ }^{6}$ the chamber cross section area is 58 times larger than the area of the coaxial injector.

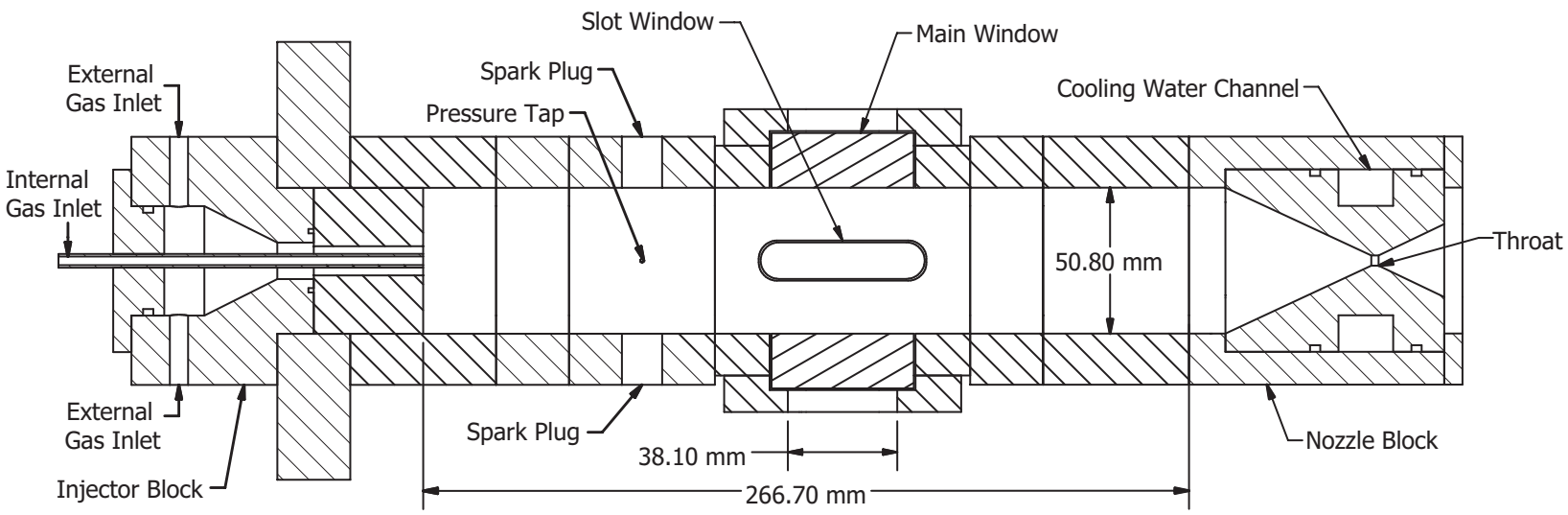

Figure 3. Schematic of the Michigan Single Element Injector Experiment.

\section{III.A. Quantitative Acetone PLIF for Nonreacting Cases}

Acetone fluorescence was excited by a Nd:YAG laser $(266 \mathrm{~nm}) \cdot{ }^{18-20}$ Fluorescence was collected between $400 \mathrm{~nm}$ and $700 \mathrm{~nm}$ with a Sony XCD-710 camera using a f/1.4 $50 \mathrm{~mm}$ Nikkor lens. The BK7 glass lens effectively blocked scattering from the $266 \mathrm{~nm}$ beam. A fraction of the beam was sent through a reference cell containing the acetone-seeded inner jet gas. Fluorescence from the reference cell was used for normalization and to correct for shot-to-shot power variations. An absorption spectroscopy measurement provided acetone mole fraction in the seeded fluid, allowing the conversion from mole fraction to mixture fraction. Corrections for sheet non-uniformity were made by normalizing each image by an image taken with the test section filled with an uniform acetone-air mixture. In addition, corrections for background scatter, dark noise, and absorption were made. ${ }^{21,22}$ A nominal 18 percent by volume acetone seeding level was used for all atmospheric cases. Acetone seeding was adjusted at other chamber pressures to maintain the same fluorescence signal level. 


\section{III.B. Qualitative OH PLIF for Reacting Cases}

$\mathrm{OH}$ fluorescence was obtained by taking the $532 \mathrm{~nm}$ beam of a Nd:YAG laser and passing it through a dye laser containing Rhodamine 590 dye to produce a yellow beam at $566 \mathrm{~nm}$ with a linewidth of $0.06 \mathrm{~cm}^{-1}$. Using a doubling crystal the $566 \mathrm{~nm}$ beam was doubled to $283 \mathrm{~nm}$. This beam excited the $Q_{1}(6)$ transition of the $A^{2} \Sigma^{+} \leftarrow X^{2} \Pi(1,0)$ band. The $283 \mathrm{~nm}$ beam with $5 \mathrm{~mJ}$ per pulse produced a sheet with a hight of 35 $\mathrm{mm}$ with a thickness of $350 \mu \mathrm{m}$. Images were taken at 5 frames per second using a Princeton Instruments PI-MAX intensified Camera with a $105 \mathrm{~mm}$ Nikkor UV lens operating at f/4.5. OH fluorescence was collected from the $\mathrm{A}-\mathrm{X}(1,1)$ and $(0,0)$ bands around $310 \mathrm{~nm}$. Images were $400 \times 300$ pixels. A 100 ns gate was used along with WG-295 and UG-11 Schott glass filters to block scattering and flame luminosity. A diagram of the $\mathrm{OH}$ system is shown in Fig. 4. Due to heating of the experimental test section, runs were limited to 18 seconds during which 15 seconds were used to collect 75 images. Two runs were conducted at every data point and window height to collect $\mathrm{OH}$ fluorescence. A third run was taken to correct for flame luminosity. Instantaneous sheet corrections were made by splitting off a portion of the sheet and imaging the sheet in a cell filled with an optically thick mixture of laser dye.

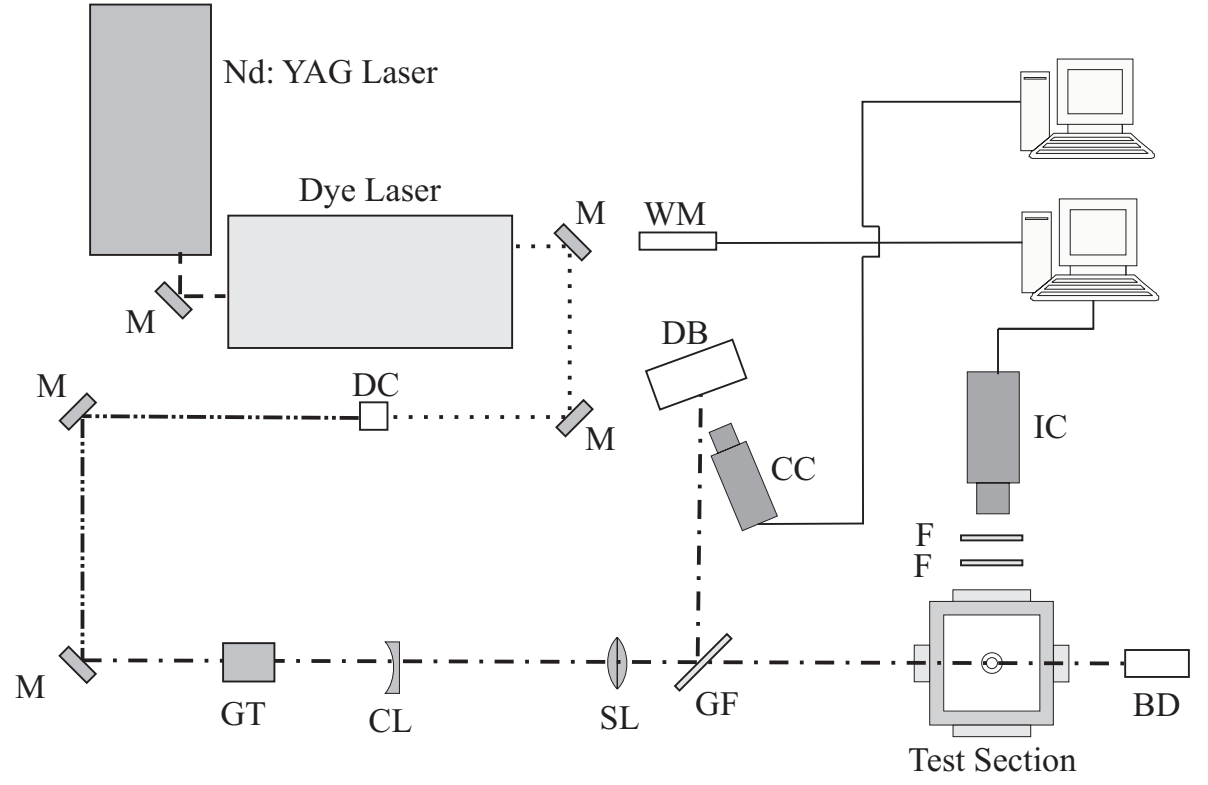

Figure 4. Laser and optical setup for qualitative OH PLIF measurements. BD - beam dump, CC - CCD camera, CL cylindrical lens, DC - doubling crystal, DB - dye cell, F - filter, GF - glass flat, GT - Galilean telescope, IC - intensified camera, M - mirror, SL - spherical lens, WM -wavelength meter. (- - - ) - $532 \mathrm{~nm},(\cdots)-566 \mathrm{~nm},(-\cdot-)-283 \mathrm{~nm}$

Calculations were performed to determine which of the OH PLIF signal contours best represents the stoichiometric contour (i.e. the flame front). Following the method outlined by Barlow and Collignon ${ }^{12}$ and using the $\mathrm{OH}$ quenching model of Paul, ${ }^{23}$ the effect of Boltzmann fraction and quenching on the $\mathrm{OH}$ signal were investigated. Simulations of counterflow flames performed using the OPDIFF code in Chemkin and the GRI-mech chemical mechanism provided representative profiles of mole fraction and temperature found in a hydrogen-oxygen and hydrogen-air turbulent coaxial jet flames. For hydrogen-oxygen these profiles were found to be independent of the strain rate over 100 to $2000 \mathrm{~s}^{-1}$, whereas the peak values where found to vary with strain rate for hydrogen-air over 100 to $1000 \mathrm{~s}^{-1}$. From these profiles, corrections for the effect of Boltzmann fraction and quenching were made to produce a simulated LIF signal. Figure 5(b) shows the effect of these phenomena for hydrogen-oxygen is to shift the peak $\mathrm{OH}$ signal towards the lean side of the flame and that the stoichiometric value occurs at $67 \%$ of the peak $\mathrm{OH}$ signal on the rich side of the flame. For hydrogen-air the stoichiometric value occurs at approximately $94 \%$ of the peak on the rich side of the flame for the strain rates investigated. These results were obtained using the adiabatic flame temperature as the reference condition as discussed by Barlow and Collignon. ${ }^{12}$ A calibration constant related to this reference condition and the PLIF system in general being unknown is assumed to be 1 . Hence the shape and location of the simulated LIF signal in Fig. 5 is accurate, while the magnitude of the signal is arbitrary. In addition this method was used to evaluate different rotational levels in the $Q_{1}$ band. It was found that 
for hydrogen-oxygen chemistry when Boltzmann fraction and quenching were considered that the $Q_{1}(6)$ transition and higher transitions such as $Q_{1}(9)$ produced nearly identical (within 1\%) signal profiles. Given the nearly identical $\mathrm{OH}$ signal profiles of these two transitions, the significantly larger laser energy of the $Q_{1}(6)$ transition was selected.

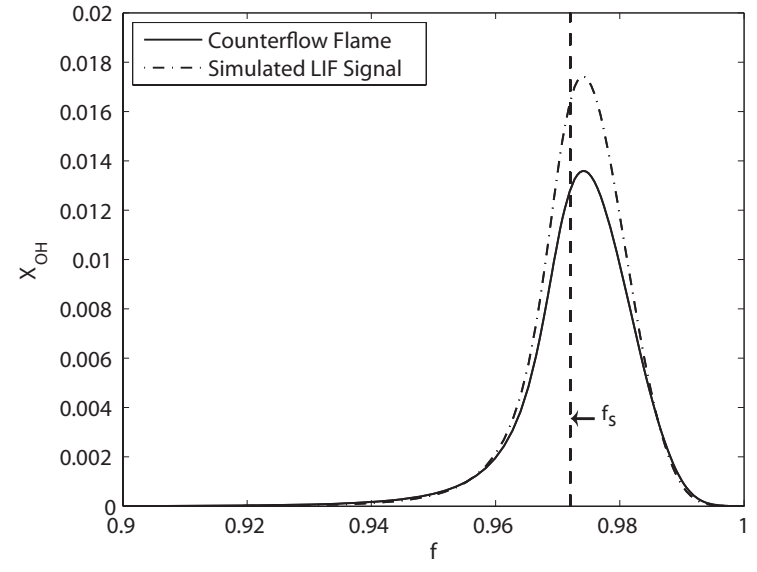

(a)

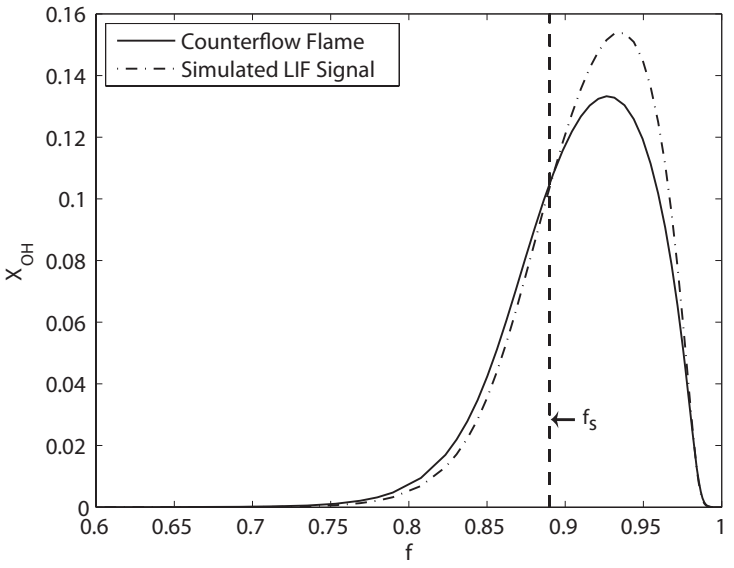

(b)

Figure 5. OH mole fraction $\left(X_{O H}\right)$ vs. mixture fraction $(f)$ for a) hydrogen-air and b) hydrogen-oxygen counterflow flame and simulated LIF signal corrected for Boltzmann fraction and quenching effects for the $Q_{1}(6)$ transition. Note the simulated LIF signal magnitude is arbitrary due to an unknown calibration constant, however shape and location are accurate.

\section{III.C. Experimental Conditions}

Test conditions were divided into nine groups. Within each group data were taken for the same diameter ratio $(S)$, chamber pressure $\left(P_{C}\right)$, and reacting or nonreacting conditions, but velocity ratio $\left(r_{u}\right)$ was varied. These groups are listed in Table 1, where i denotes inner jet properties and e denotes outer jet properties and $T_{P}$ is the thickness of the injector post. The Reynolds number $(R e)$ is based on a theoretical jet with external jet fluid properties and a velocity that provides the same total momentum flux as the actual coaxial jet,

$$
\operatorname{Re}=\frac{\rho_{e} d_{e} u_{e}}{\mu_{e}} \times\left[1-\left(\frac{2 T_{P}+d_{i}}{d_{e}}\right)^{2}+\frac{1}{M}\left(\frac{d_{i}}{d_{e}}\right)^{2}\right]^{\frac{1}{2}} .
$$

Chamber pressure was varied to check for Reynolds number independence in both the reacting and nonreacting cases.

Table 1. Experimental data groups

\begin{tabular}{|c|c|c|c|c|c|c|c|c|c|c|}
\hline Group & $\mathrm{S}$ & $\mathrm{r}_{\mathrm{u}}$ & $\mathrm{d}_{\mathrm{e}}, \mathrm{mm}$ & $\mathrm{d}_{\mathrm{i}}, \mathrm{mm}$ & $\mathrm{T}_{\mathrm{P}}, \mathrm{mm}$ & $\mathrm{X}_{\mathrm{s}}$ & $\operatorname{Re}$ & $\mathrm{P}_{\mathrm{C}}$, atm & Outer/Inner & Reacting \\
\hline G1 & 0.132 & $5.0-1.1$ & 7.5 & 3.0 & 0.89 & 0.52 & $15,000-14,000$ & 3.9 & He-Air & No \\
\hline G2 & 0.133 & $5.0-1.1$ & 7.5 & 3.0 & 0.89 & 0.52 & $23,000-21,000$ & 5.4 & He-Air & No \\
\hline G3 & 0.063 & $8.0-2.5$ & 6.7 & 3.4 & 0.72 & 0.33 & $53,000-32,000$ & 4.4 & $\mathrm{H}_{2}-\mathrm{O}_{2}$ & Yes \\
\hline G4 & 0.063 & $5.0-2.5$ & 6.7 & 3.4 & 0.72 & 0.33 & $72,000-55,000$ & 8.4 & $\mathrm{H}_{2}-\mathrm{O}_{2}$ & Yes \\
\hline G5 & 0.070 & $3.0-2.1$ & 5.8 & 3.4 & 0.72 & 0.70 & $25,000-22,000$ & 4.8 & $\mathrm{H}_{2}$-Air & Yes \\
\hline G6 & 0.118 & $10-1.1$ & 7.5 & 3.0 & 0.89 & 0.52 & $4,100-3,500$ & 1.0 & He-Air & No \\
\hline G7 & 0.058 & $10-2.0$ & 6.7 & 3.4 & 0.72 & 0.32 & $9,200-4,700$ & 1.0 & $\mathrm{H}_{2}$-Air & No \\
\hline G8 & 0.462 & $5.1-2.5$ & 7.5 & 3.0 & 0.89 & 0.65 & $45,000-23,000$ & 1.0 & $\mathrm{CH}_{4}$-Air & No \\
\hline G9 & 0.063 & 6.3-1.9 & 6.7 & 3.4 & 0.72 & 0.33 & $6,800-4,300$ & 1.0 & $\mathrm{H}_{2}-\mathrm{O}_{2}$ & Yes \\
\hline
\end{tabular}




\section{Results}

Average and instantaneous mixture fraction fields for nonreacting coaxial jets were obtained using acetone PLIF. A representative set of images from group G2 with varying velocity ratios $\left(r_{u}\right)$ are presented in Fig. 7, where the inner jet fluid is acetone seeded air and the outer fluid is helium. The white line marks the contour where $f_{S}=0.89$, which is the stoichiometric value for hydrogen-oxygen chemistry. Average images show the flow field to be symmetrical and the growth of the external shear layer between the ambient fluid is clearly visible. Due to the confined nature of the flow, the ambient fluid (between the jet and the wall) is the result of the complete mixing of both jet fluids and hence changes from case-to-case. Since the mixing occurs primarily in the near field, the measured values of $L_{S}$ are independent of the ambient fluid. It is important to note that all cases are run fuel rich so no mixing with the ambient fluid is needed. Instantaneous images of the mixture fraction field show the turbulent nature of the flow field along with the break up of the tip and the formations of pockets. The growth of $L_{S}$ with decreasing velocity ratio can clearly be seen. Additional nonreacting coaxial jet data and analysis were previously presented by the authors. ${ }^{6}$

Instantaneous $\mathrm{OH}$ images were obtained for hydrogen-oxygen and hydrogen-air turbulent coaxial jet flames. Figures 8(d)-(f) show instantaneous $\mathrm{OH}$ images corresponding to three cases with different $r_{u}$ values of group G3, whereas Fig. 9 shows a single instantaneous image with a velocity ratio of approximately 3.3 from each reacting data group. From these images, it is clear that $\mathrm{OH}$ forms thin layers compared to the large diffuse regions of $\mathrm{OH}$ usually seen in $\mathrm{OH}$ PLIF images. Thin layers form in this configuration since the oxidizer is encased in a hot fuel rich envelope which consumes any $\mathrm{OH}$ which diffuses out. Figures 9(b)-(d) are hydrogen-oxygen flames at increasing pressure and hence increasing Reynolds number. These images show a decrease in the thickness of the $\mathrm{OH}$ layers with pressure which is expected from the diffusion reaction rate balance which sets reaction layer thickness. Also the hydrogen-air case, Fig. 9(a), being of similar pressure to the middle hydrogen-oxygen case, has the thinnest layers. This thinness is due to the reduced heat release in the hydrogen-air reaction which results in reduced peak $\mathrm{OH}$ concentrations and a narrowed $\mathrm{OH}$ vs mixture fraction profile compared to hydrogen-oxygen which is illustrated in Fig. 5.

The structures of turbulent diffusion flames have been described in a number of studies. ${ }^{11,24,25}$ These same structures can be seen in these inverted coaxial turbulent diffusion flames. Close to the jet, exit laminar-like regions exist where the heat release has suppressed the turbulence. Small turbulent wrinkles (structures) grow from this region and increase in size as they move downstream eventually breaking though the high viscosity layer of the flame bringing entrained external jet fluid which can lower the stoichiometry enough in some cases to lead to localized extinction. The local extinctions then cause the break up of the flame tip and the formation of flame pockets. Mungal et al. ${ }^{24,25}$ suggest that the two dominant instability modes in diffusion flames are axi-symmetric and helical which correspond to symmetric doughnut shapes and an anti-symmetric sinuous mode in the $\mathrm{OH}$ layers respectively. Both types of instabilities can be seen in the reacting cases, but additional analysis is needed to quantify the relative occurrence of each mode. Mungal et al. ${ }^{25}$ also suggests that these structures and flame tip separation occur in a quasi periodic manner however this is impossible to verify with the present data since it is not time resolved.

To test the scaling law for coaxial jets (Eq. (5)) and the equivalence principle of Tacina and Dahm, ${ }^{13}$ $L_{S}$ for reacting cases were calculated in the following manner. Given the relatively thin nature of the $\mathrm{OH}$ signal and the counterflow flame results discussed in Section III.B, the local peak signal was taken to be a good indicator of the stoichiometric contour and local thresholding was used to create an instantaneous flame contour. The use of the peak signal instead of $67 \%$ of the peak signal (which is the true stoichiometric contour) for hydrogen-oxygen results in an approximate $0.3 \mathrm{~mm}$ shift in the stoichiometric contour or a $0.6 \%$ error in $L_{S}$. The error is even less for the hydrogen-air case since the stoichiometric value occurs at $94 \%$ of the peak $\mathrm{OH}$ signal. These instantaneous flame contours were then averaged. Figures 8(a)-(c) show a thin base region (corresponding to the small turbulent wrinkles) whereas downstream the flame brush is broad and nearly uniform. $L_{S}$ was determined from the average images and is defined as the most probable location of the center of the thin $\mathrm{OH}$ layers on the centerline. Changes in this definition result in a minimal vertical shift in the data, but all slopes remain constant. In addition, this definition was found to correspond well with measurements made from the instantaneous images. Results show that the reacting cases have much larger values of $L_{S}$ than the nonreacting cases for comparable $M$ values. The increase in $L_{S}$ is due to the fact that mass entrainment in all reacting shear flows is considerably less than in their nonreacting counterparts due to the lower density created by combustion.

Stoichiometric mixture length values are plotted in Fig. 6a) and c) against $M$ and $X_{S}$. Data were divided into two plots based on the chamber pressure. This division was done because it was found that the one 
atmosphere data collapses to a line with one slope and the elevated pressure data collapses to a line with a larger slope when plotted against $M^{1 / 2}$. This trend is opposite to what one would expect if the Reynolds number is the reason for the different slopes. At higher Reynolds numbers one would expect slightly better mixing (smaller $L_{S}$ ), but the opposite is seen. Reacting and nonreacting data were taken at two elevated chamber pressures to test if this effect continued as the pressure was raised. All elevated pressure data were found to collapse to a curve within experimental error. This phenomenon was previously related to a delay in the spreading of the outer shear layer for atmospheric nonreacting cases, however this increased entrainment is not fully understood and is still under investigation.

Figures 6a) and c) show the the use of the momentum ratio (M) causes the values of $L_{S}$ to collapse to a single curve for nonreacting and a single curve for reacting data. The reacting data has a larger slope and lies above the nonreacting due to heat release as previously explained. It is surprising however that in Fig. 6c) the hydrogen-air data (group G5) aligns with the hydrogen-oxygen data (groups G3 \& G4) given the difference in heat release even with the change in stoichiometry accounted for with $X_{S}$.

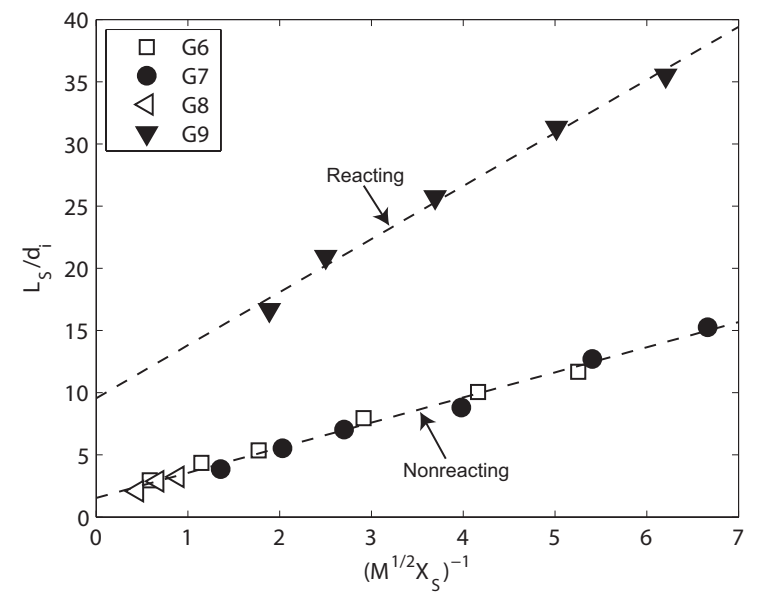

(a) Atmospheric pressure momentum ratio scaling

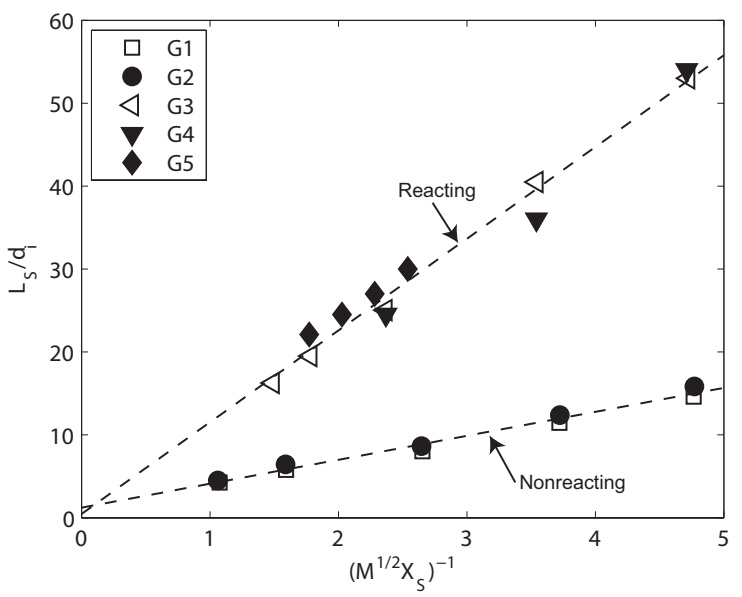

(c) Elevated pressure momentum ratio scaling

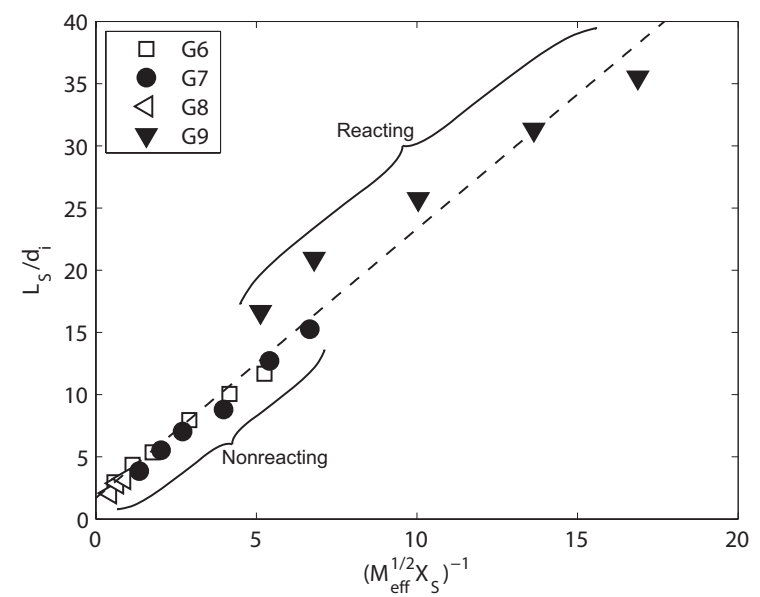

(b) Atmospheric pressure effective momentum ratio scaling

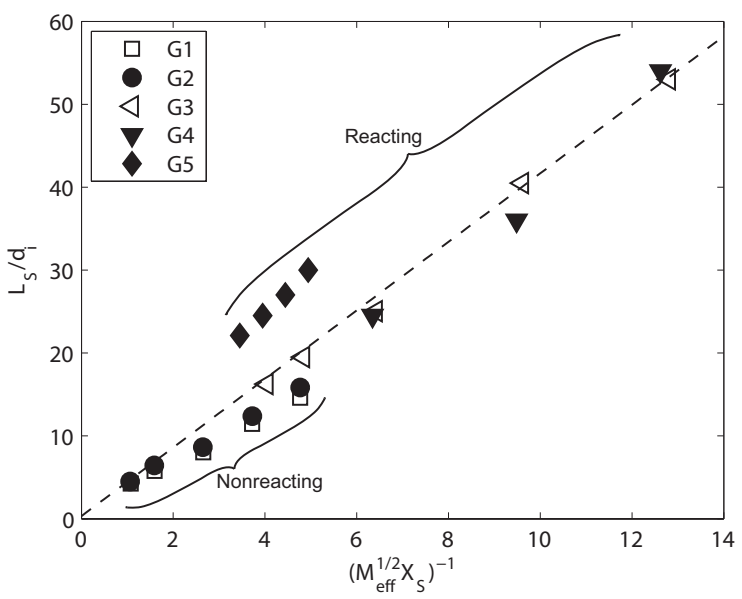

(d) Elevated pressure effective momentum ratio scaling

Figure 6. Stoichiometric mixing lengths for nonreacting coaxial jets and coaxial diffusion flames plotted for atmospheric pressure data a),b) and elevated pressure data c), d) against the momentum ratio scaling a), c) and the effective momentum ratio scaling b),d) based on the equivalence principle of Tacina and Dahm. ${ }^{13}$ See Table 1 for explanation of data groups.

In an effort to test the theory of Tacina and Dahm ${ }^{13}$ which accounts for heat release, $L_{S}$ values are replotted in Figs. 6b) and d) with $M$ replaced by $M_{\text {eff }}$. It can be seen that whereas the nonreacting data and the hydrogen-oxygen reacting data (groups G3 and G4) collapse to a single curve, the effect of heat release for the hydrogen-air data (group G5) is under predicted. If the equivalence principle calculations (Fig. 2) are reexamined, it is clear for the hydrogen-air case the effective values of temperature and molecular weight accurately predict the temperature and molecular weight profiles of the inner jet fluid which in turn 
accurately predict the density profile. However, in the case of hydrogen-oxygen the equivalence principle over predicts the inner jet temperature profile due to the nonlinearity in the equilibrium state relation caused by dissociation effects which results in an under prediction of the inner fluid density. This result means that it would be expected for the equivalence principle to over predict the effects of heat release for hydrogen-oxygen. Thus it is believed that gas dissociation which is not considered by the Tacina-Dahm analysis explains why the collapse of the hydrogen-oxygen data is better then hydrogen-air and reiterates that the Tacina and Dahm equivalence principle under predicts the effect of heat release in turbulent coaxial jet flames.

An assumption of both the cold flow scaling relation (Eq. (5)) and the Tacina and Dahm equivalence principle is that buoyancy effects are negligible. To quantify the effects of buoyancy in the present configuration, the Richardson number based method of Becker and Yamazaki. ${ }^{26}$ was applied for reacting cases whereas the method of Favre-Marinet and Schettini ${ }^{3}$ was used for nonreacting cases. From these methods it was found that all nonreacting and reacting cases were momentum-dominated and that buoyancy effects were small.

As mentioned in Section III.B images are collected over a 15 second interval due to thermal loading. During this time, the pressure in the chamber slightly increases and the temperature of the ambient fluid also increases. To explore if this increase in temperature and pressure has any effect on $L_{S}$, images from group G3 were divided into two groups based on their location in a run with 5 images in the middle of the run thrown out. These images were then processed in the same manner as previously mentioned. Results showed percent differences in calculated values of less then $3 \%$, however a marginal increase in $L_{S}$ towards the end of runs was observed. This analysis further confirms that buoyancy plays a miner role since any increase in ambient fluid temperature would decrease buoyancy resulting in shorter flames.

\section{Conclusions}

Average and instantaneous mixture fraction fields were obtained for nonreacting turbulent coaxial jets using acetone PLIF and compared to $\mathrm{OH}$ radical images obtained in reacting turbulent coaxial jet flames for hydrogen-oxygen and hydrogen-air reactions utilizing OH PLIF. To aid in the comparison of reacting and nonreacting cases and to explore the effects of the momentum ratio $(M)$ on mixing, stoichiometric mixing lengths $\left(L_{S}\right)$ were measured. For nonreacting cases $L_{S}$ was obtained directly from the mixture fraction field. Given that the $\mathrm{OH}$ signal was contained in thin layers due to the inner jet of oxidizer being surrounded by an outer jet of hydrogen, local thresholding was used to determine instantaneous flame fronts. When averaged, the instantaneous flame fronts yielded a value of $L_{S} . L_{S}$ was found to scale with $M$ for all data groups, however scaling constants differed for reacting and nonreacting cases and for cases taken at atmospheric chamber pressure versus elevated chamber pressure. For both reacting and nonreacting data, cases taken at atmospheric pressure were found to have shorter values of $L_{S}$ than the elevated pressure cases. This is opposite to what would be expected based on a Reynolds number arguments since increasing Reynolds number results in larger $L_{S}$. Increasing the chamber pressure and hence the Reynolds number from 3.9 to 8.4 atmospheres was shown to have no further effect on $L_{S}$. Curves of $L_{S}$ for reacting data groups at all chamber pressures were found to be higher and have larger slopes then the corresponding nonreacting cases. This is due to the decrease in mass entrainment caused by heat release. To account for heat release, the equivalence principle of Tacina and Dahm ${ }^{13}$ was applied where the density of the outer jet fluid is replaced with a smaller effective density. This method was found to work reasonably well, but slightly under predicted the effect of heat release for hydrogen-air and hydrogen-oxygen reactions. The better collapse of the hydrogen-oxygen data was shown to be due the the equivalence principle under predicting the inner jet density profile due to nonlinearities in the equilibrium state relation caused by dissociation effects. Additional work is needed to improve methods to more accurately account for heat release effects in turbulent coaxial jet flames and to account for differences in the entrainment rate as the chamber pressure is varied.

\section{Acknowledgments}

This work is supported by the NASA Constellation University Institutes Program (CUIP) under grant NCC3-989 jointly funded by NASA and DOD, with Claudia Meyer as the project manager. 


\section{References}

${ }^{1}$ Dahm, W. J. A., Frieler, C. E., and Tryggvason, G., "Vortex Structure and Dynamics in the Near Field of a Coaxial Jet," Journal of Fluid Mechanics, Vol. 241, 1992, pp. 371-402.

${ }^{2}$ Villermaux, E. and Rehab, H., "Mixing in Coaxial Jets," Journal of Fluid Mechanics, Vol. 425, 2000, pp. 161-185.

${ }^{3}$ Favre-Marinet, M. and Schettini, E. B. C., "The Density Field of Coaxial Jets with Large Velocity Ratio and Large Density Differences," International Journal of Heat and Mass Transfer, Vol. 44, 2001, pp. 1913-1924.

${ }^{4}$ Champagne, F. H. and Wygnanski, I. J., "An Experimental Investigation of Coaxial Turbulent Jets," International Journal of Heat and Mass Transfer, Vol. 14, 1971, pp. 1445-1464.

${ }^{5}$ Ko, N. W. M. and Kwan, A. S. H., "The Inital Region of Subsonic Coaxial Jets," Journal of Fluid Mechanics, Vol. 73, 1976, pp. 305-332.

${ }^{6}$ Schumaker, S. A. and Driscoll, J. F., "Mixing Lengths of Coaxial Jets in a Rocket Combustor Configuration using Acetone PLIF," 43rd Joint Propulsion Conference, Cincinnati, OH, 2007, AIAA Paper 2007-5590.

${ }^{7}$ Moser, M. D., Merenich, J. J., Pal, S., and Santoro, R. J., "OH-Radical Imaging and Velocity Field Measurements in a Gaseous Hydrogen/Oxygen Rocket," 29th Joint Propulsion Conference, Monterey, CA, 1993, AIAA Paper 1993-2036.

${ }^{8}$ Yeralan, S., Pal, S., and Santoro, R. J., "Experimental Study of Major Species and Temperature Profiles of Liquid Oxygen/Gaseous Hydrogen Rocket Combustion," Journal of Propulsion and Power, Vol. 14, 2001, pp. 788-793.

${ }^{9}$ Ditaranto, M., Sautet, J. C., and Samaniego, J. M., "Structural Aspects of Coaxial Oxy-fuel Flames," Experiments in Fluids, Vol. 30, 2001, pp. 253-261.

${ }^{10}$ Nicoli, C. and Haldenwang, P., "Combustion of Gaseous Coflow Jets," Combustion Science and Technology, Vol. 175, 2003, pp. 1143-1163.

${ }^{11}$ Sautet, J. C., Salentey, L., and DiTaranto, M., "Large-Scale Turbulent Structures in Non-premixed, Oxygen Enriched Flames," International Communications in Heat and Mass Transfer, Vol. 28, 2001, pp. 277-287.

${ }^{12}$ Barlow, R. S. and Collignon, A., "Linear LIF Measurments of OH in Nonpremixed Methane-Air Flames: When are Quenching Corrections Unnecessary," 29th Aerospace Sciences Meeting, Reno, NV, 1991, Paper AIAA-1991-0179.

${ }^{13}$ Tacina, K. M. and Dahm, W. J. A., "Effects of Heat Release on Turbulent Shear Flows. Part 1. A General Equivalence Principle for Non-Buoyant Flows and its Application to Turbulent Jet Flames," Journal of Fluid Mechanics, Vol. 415, 2000, pp. 23-44.

${ }^{14} \mathrm{Dahm}$, W. J. A., "Effects of Heat Release on Turbulent Shear Flows. Part 2. Turbulent Mixing Layers and the Equivalence Principle," Journal of Fluid Mechanics, Vol. 540, 2005, pp. 1-19.

${ }^{15}$ Schumaker, S. and Driscoll, J. F., "Coaxial Turbulent Jet Flames: Scaling Relations for Measured Stoichiometric Mixing Lengths," To appear in Proceedings of the Combustion Institute, Vol. 32, 2009.

${ }^{16}$ HIll, B. J., "Measurment of Local Entrainment Rate in The Initial Region of Axisymmetric Turbulent Air Jets," Journal of Fluid Mechanics, Vol. 51, 1972, pp. 773-779.

${ }^{17}$ Schumaker, S. A. and Driscoll, J. F., "Rocket Combustion Properties for Coaxial Injectors Operated at Elevated Pressures," 42nd Joint Propulsion Conference, Sacramento, CA, 2006, AIAA Paper 2006-4704.

${ }^{18}$ Yuen, L. S., Peters, J. E., and Lucht, R. P., "Pressure Dependence of Laser-Induced Fluorescence from Acetone," Applied Optics, Vol. 36, 1997, pp. 3271-3277.

${ }^{19}$ Lozano, A., Yip, B., and Hanson, R. K., "Acetone: a Tracer for Concentration Measurments in Gaseous Flows by Planar Laser-Induced Fluorescence," Experiments in Fluids, Vol. 13, 1992, pp. 369-376.

${ }^{20}$ Thurber, M. C. and Hanson, R. K., "Pressure and Composition Dependences of Acetone Laer-Induced Fluorescence with excitation at 248, 266, 308nm," Applied Physics B, Vol. 69, 1999, pp. 229-240.

${ }^{21}$ van Cruyningen, I., Lozano, A., and Hanson, R. K., "Quantitative Imaging of Concentration by Planar Laser-Induced Fluorescence," Experiments in Fluids, Vol. 10, 1990, pp. 41-49.

${ }^{22}$ Clemens, N. T., "Encyclopedia of Imaging Science and Technology," Encyclopedia of Imaging Science and Technology, edited by J. P. Hornak, John Wiley and Sons, New York, 2002, pp. 390-419.

${ }^{23}$ Paul, P. H., "A Model for Temperature-Dependent Collisional Quenching of $\mathrm{OH} A^{2} \Sigma^{+}$," Journal of Quantitative Spectroscopy and Radiative Transfer, Vol. 51, 1994, pp. 511-524.

${ }^{24}$ Mungal, M. G. and O’Neil, J. M., "Visual Obervations of a Turbulent Diffusion Flame," Combustion and Flame, Vol. 78, 1989, pp. 377-389.

${ }^{25}$ Mungal, M. G., Karasso, P., and Lozano, A., "The Visible Structure of Turbulent Jet Diffusion Flames Large-Scale Organization and Flame Tip Oscillation," Combustion Science and Technology, Vol. 76, 1991, pp. 165-185.

${ }^{26}$ Becker, H. A. and Yamazaki, S., "Entrainment, Momentum Flux and Temperature in Vertical Free Turbulent Diffusion Flames," Combustion and Flame, Vol. 33, 1978, pp. 123-149. 


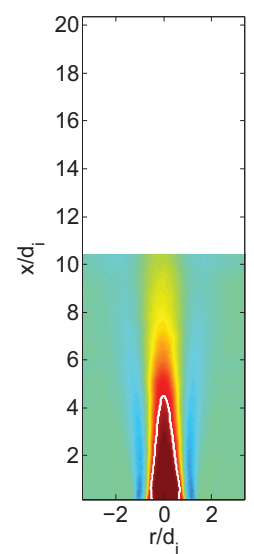

(a) $r_{u}=5.0$

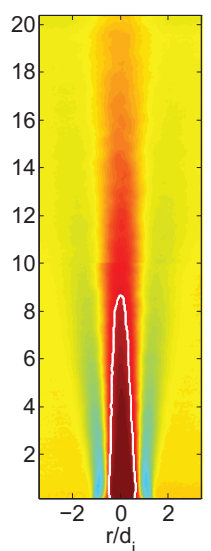

(b) $r_{u}=2.0$

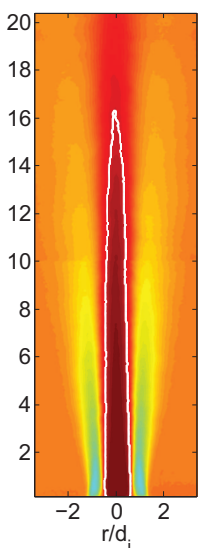

(c) $r_{u}=1.1$

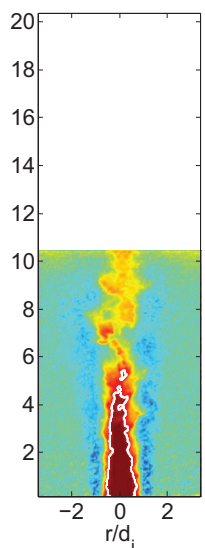

(d) $r_{u}=5.0$

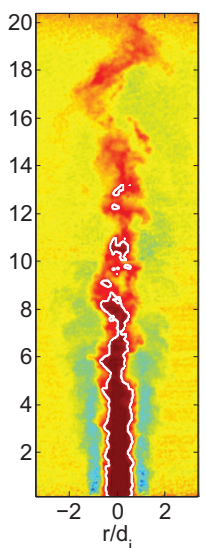

(e) $r_{u}=2.0$

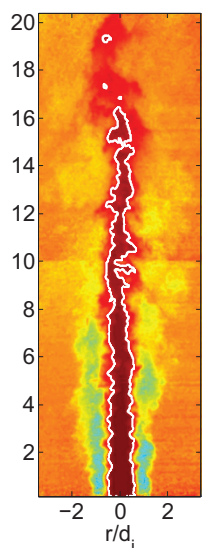

(f) $r_{u}=1.1$

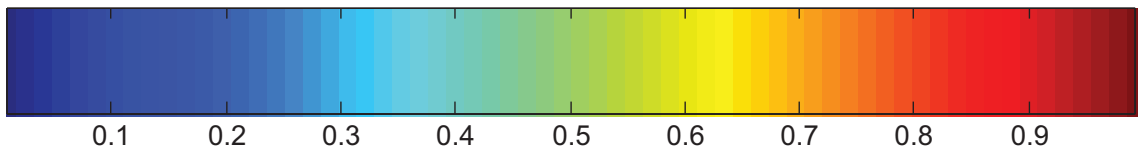

Figure 7. Time averaged a)-c) and instantaneous d)-f) mixture fraction fields of nonreacting turbulent coaxial jets for varying velocity ratios $\left(r_{u}\right)$ obtained using acetone PLIF. Inner jet fluid is acetone seeded air and annular jet fluid is helium, corresponding to group G2. Images from two window locations, $(\mathrm{x} / \mathrm{d}=0 \mathrm{0-10}$ and $\mathrm{x} / \mathrm{d}=10-20)$ are superimposed.

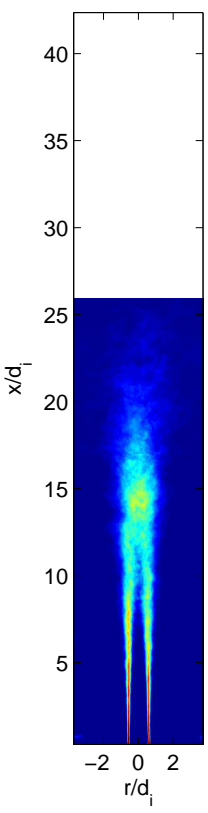

(a) $r_{u}=8.0$

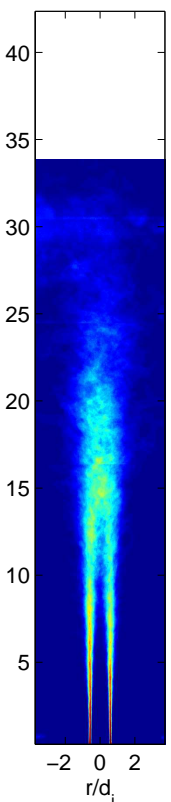

(b) $r_{u}=6.7$

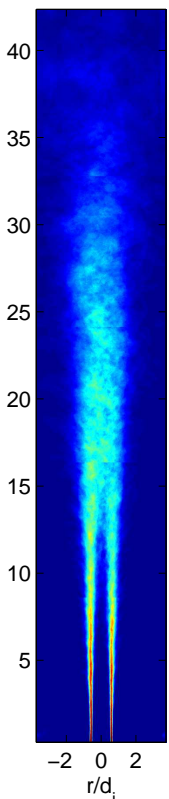

(c) $r_{u}=5.0$

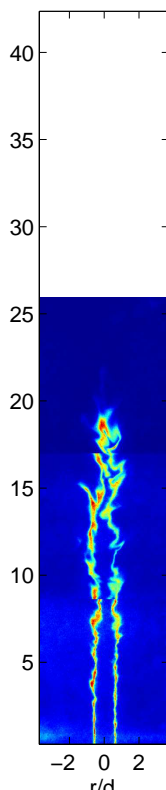

(d) $r_{u}=8.0$

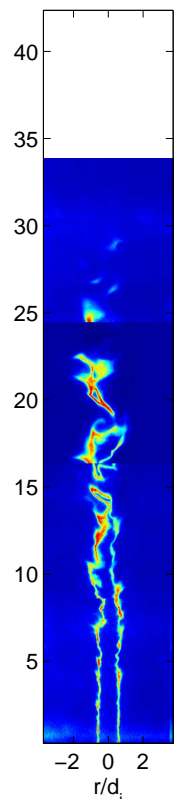

(e) $r_{u}=6.7$

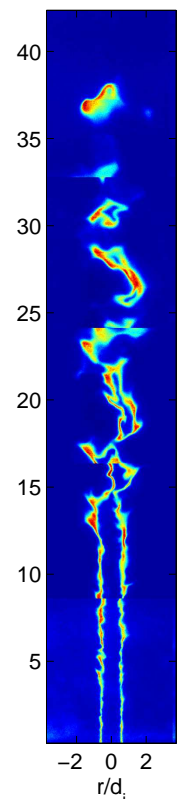

(f) $r_{u}=5.0$

Figure 8. Time averages of thresholded instantaneous $\mathrm{OH}$ contours a)-c) and instantaneous OH contours d)-f) for hydrogen, annular jet fluid, and oxygen, inner jet fluid, turbulent coaxial jet flames with varying velocity ratio $\left(r_{u}\right)$ values (Group G3). Images from five window locations are superimposed. 


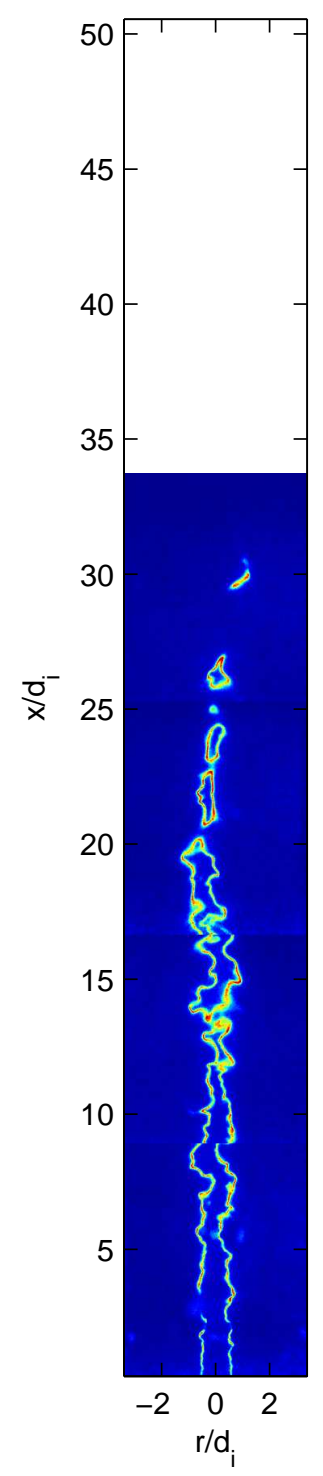

(a) Air, $\mathrm{P}_{\mathrm{C}}=4.8$, G5

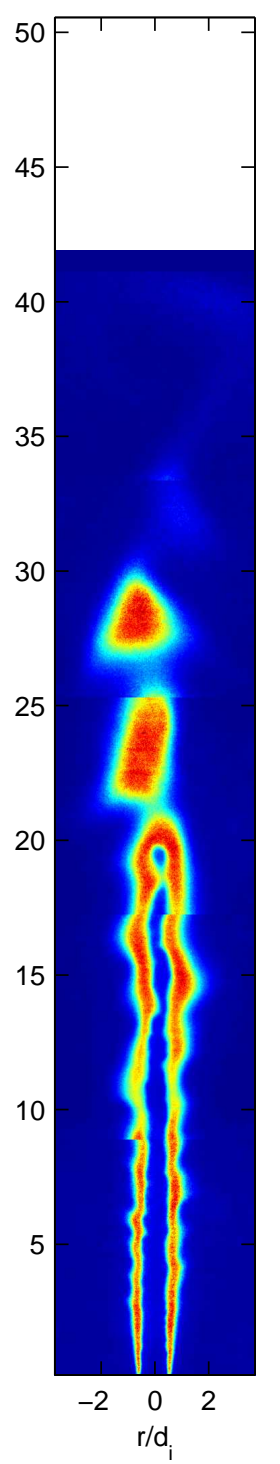

(b) $\mathrm{O}_{2}, \mathrm{P}_{\mathrm{C}}=1.0$, G9

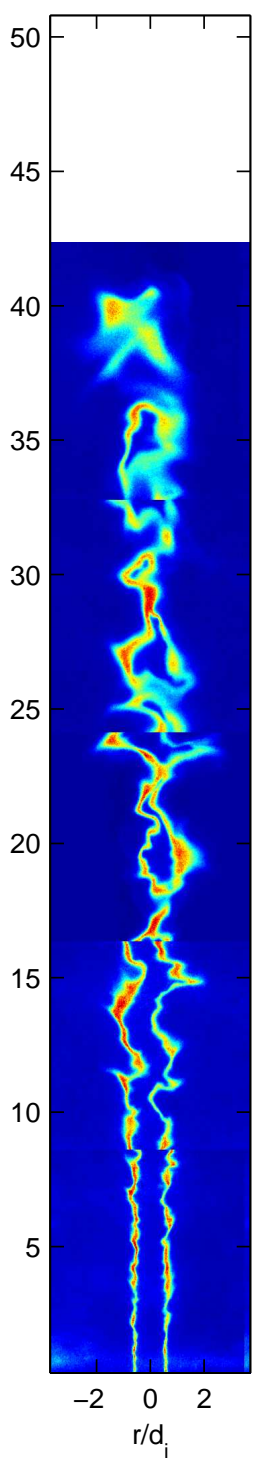

(c) $\mathrm{O}_{2}, \mathrm{P}_{\mathrm{C}}=4.4, \mathrm{G} 3$

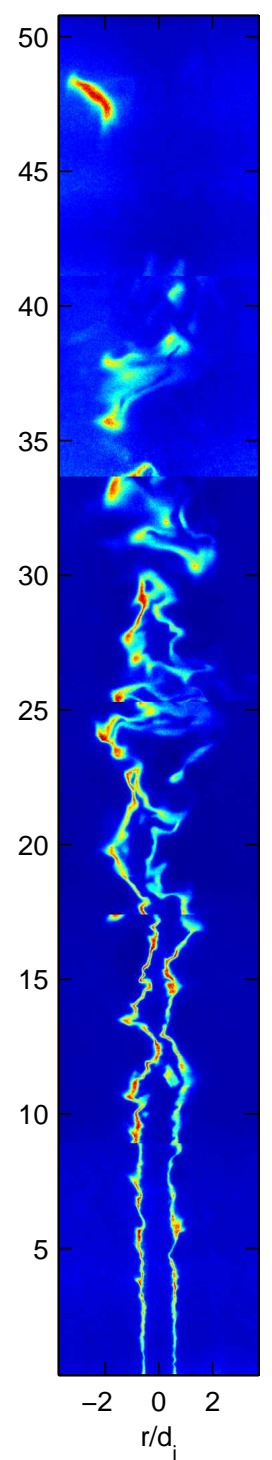

(d) $\mathrm{O}_{2}, \mathrm{P}_{\mathrm{C}}=8.4, \mathrm{G} 4$

Figure 9. Instantaneous OH Images for hydrogen-oxygen b)-d) and hydrogen-air a) turbulent coaxial jet flames at various chamber pressures with $r_{u} \approx 3.3$. Breaks in the $\mathrm{OH}$ layers in the base region of image a) are due to water on the windows and not extinction. 